\title{
Late onset asymptomatic pancreatic neuroendocrine tumor - A case report on the phenotypic expansion for MEN1
}

\author{
Charu Kaiwar ${ }^{1}$, Sarah K. Macklin², Jennifer M. Gass ${ }^{3,4}$, Jessica Jackson², Eric W. Klee ${ }^{5,6,7}$, Stephanie L. Hines ${ }^{8}$,
} John A. Stauffer ${ }^{9}$ and Paldeep S. Atwal ${ }^{2^{*}}$ (D)

\begin{abstract}
Background: Multiple endocrine neoplasia type 1 (MEN1) is a hereditary cancer syndrome associated with several endocrine as well as non-endocrine tumors and is caused by mutations in the MEN1 gene. Primary hyperparathyroidism affects the majority of MEN1 individuals by age 50 years. Additionally, MEN1 mutations trigger familial isolated hyperparathyroidism. We describe a seemingly unaffected 76-year-old female who presented to our Genetics Clinic with a family history of primary hyperparathyroidism and the identification of a pathogenic MEN1 variant.

Case Presentation: The patient was a 76 year-old woman who appeared to be unaffected. She had a family history of a known MEN1 pathogenic variant. Molecular testing for the known MEN1 mutation C.1A > G, as well as, biochemical testing, MRI of the brain and abdomen were all performed using standard methods. Molecular testing revealed our patient possessed the MEN1 pathogenic variant previously identified in her two offspring. Physical exam revealed red facial papules with onset in her seventies, involving her cheeks, nose and upper lip. Formerly, she was diagnosed with rosacea by a dermatologist and noted no improvement with treatment. Clinically, these lesions appeared to be facial angiofibromas. Brain MRI was normal. However, an MRI of her abdomen revealed a $1.5 \mathrm{~cm}$ lesion at the tail of the pancreas with normal adrenal glands. Glucagon was mildly elevated and pancreatic polypeptide was nearly seven times the upper limit of the normal range. The patient underwent spleen sparing distal pancreatectomy and subsequent pathology was consistent with a well-differentiated pancreatic neuroendocrine tumor (pNET).
\end{abstract}

Conclusions: Age-related penetrance and variable expressivity are well documented in families with MEN1. It is thought that nearly all individuals with MEN1 manifest disease by age 40. We present a case of late-onset MEN1 in the absence of the most common feature, primary hyperparathyroidism, but with the presence of a pNET and cutaneous findings. This family expands the phenotype associated with the c.1A > G pathogenic variant and highlights the importance of providing comprehensive assessment of MEN1 mutation carriers in families that at first blush may appear to have isolated hyperparathyroidism.

\section{Background}

Multiple endocrine neoplasia 1 (MEN1) or Wermer's Syndrome is an autosomal dominant disorder caused by germ line mutations in the MEN1 gene located in the region 11q13.1 [1]. MEN1 is a tumor suppressor gene that encodes for the protein, Menin. This 610 amino acid protein is localized within the nucleus, has two functional nuclear localization signals and represses JunD mediated transcription. It is expressed in a variety

\footnotetext{
* Correspondence: Atwal.Paldeep@mayo.edu

2Department of Clinical Genomics, Mayo Clinic, Jacksonville, FL 32224, USA

Full list of author information is available at the end of the article
}

of tissues and is conserved across species from Drosophila to Homo sapiens. Menin is proposed to act as a tumor suppressor, as MEN1-associated tumors follow Knudson's "two-hit hypothesis" [2, 3]. MEN1 is characterized by the presence of a combination of tumors of the parathyroid glands, anterior pituitary, and Islet cells of the pancreas. In addition, tumors of the adrenal cortex, facial angiofibromas, carcinoid tumors, collagenous tumors, and lipomatous tumors have also been described. A clinical diagnosis of MEN1 is made in cases where there is occurrence of two or more primary MEN1 tumor types, or where there is one of the MEN1-associated tumors and a 
family member with a clinical diagnosis of MEN1. About 10-20\% patients with familial MEN1 do not have a detectable pathogenic variant in the MEN1 gene [4]. The MEN1 phenotype is highly variable even within families and no genotype-phenotype correlations have been made to date [5]. The clinical presentation and age of diagnosis vary by the type of tumor. Primary hyperparathyroidism is the most common first presentation in patients with MEN1, followed by Pancreatic Neuroendocrine Tumors (pNET) and Pituitary tumors (PIT) [6]. In fact, primary hyperparathyroidism is diagnosed in a majority of patients before the diagnosis of MEN1. The age of diagnosis varies from the first to the fifth decade, median age being 37 years for patients with MEN1 pathogenic variants and 55 years for patients without MEN1 pathogenic variants [6].

In this report we describe a case of MEN1 where diagnosis of hyperparathyroidism in family members prompted the 76 years old asymptomatic proband to seek a genetics referral. The report expands on the phenotype of this syndrome and also highlights the phenotypic variability associated with it.

\section{Case Presentation}

A 76 year- old female was referred to our genetics clinic due to a family history of MEN1 in two children and a familial pathogenic variant in MEN1. She did not have a diagnosis of any of the "three P" tumors classic to MEN1 parathyroid, pituitary or pancreas. Her past medical history was significant for hypothyroidism and osteoporosis, for which she was being appropriately treated with levothyroxine and intermittent bisphosphate replacement. She also had a history of lymphoma, which was treated with Cyclophosphamide, Hydroxy doxorubicin, Vincristine (Oncovin), and Prednisone (CHOP) regimen. Surgical history was notable for a cholecystectomy. A complete physical examination revealed no other abnormalities, except for the presence of red papules on the cheeks, nose, vermillion border of the upper lip and chin. The patient reported that these papules had been present for the past 4-5 years. On closer examination by the geneticist, these papules were diagnosed as facial angiofibromas (Fig. 1). Her family history was significant for two daughters with MEN1 diagnosed at 48 and 53 years of age and a son with hypercalcemia and elevated parathyroid hormone levels
(Fig. 2). Following standard practice guidelines for firstdegree relatives of patients with MEN1, she was offered targeted genetic testing for the known familial pathogenic variant in $M E N 1$. Screening for endocrine tumors was also performed with MRIs of the head and abdomen (for pituitary and pancreatic tumors). Plasma $\mathrm{Ca}^{++}$, parathyroid hormone levels (PTH), prolactin and Insulin like Growth Factor (IGF-1) levels, as well as fasting gastrointestinal tract hormone profile, (that includes measurement of gastrin, glucagon, vasointestinal polypeptide, pancreatic polypeptide, chromogranin $\mathrm{A}$, and insulin with fasting glucose levels) were also performed.

Genetic testing was positive for the familial pathogenic variant $M E N 1$ c.1A > G, that results in the loss of the translation initiation codon. The imaging revealed an solitary enhancing, partially cystic pancreatic tail lesion suggestive of a neuroendocrine tumor (Fig. 3) measuring $1.1 \times 1.3 \times 1.5 \mathrm{~cm}$. Endoscopic ultrasound was then performed which confirmed the lesion in the tail of the pancreas and no other lesions were identified after thorough inspection of the entire pancreas. Laboratory tests revealed elevated pancreatic polypeptide of $2210 \mathrm{pg} / \mathrm{mL}$, while Chromogranin A was normal at $52 \mathrm{ng} / \mathrm{mL}$. The gastrointestinal hormone profile was significant only for slightly elevated glucagon at $89 \mathrm{pg} / \mathrm{mL}$. Given the isolated pancreatic lesion, the patient was considered a candidate for surgery. She underwent an uncomplicated laparoscopic spleen-preserving distal pancreatectomy and discharged the next day. Pathology confirmed it to be a $1.4 \times 1.0 \mathrm{~cm}$ well-differentiated neuroendocrine tumor, positive for keratin, synaptophysin and chromogranin stains. Regional lymph nodes were negative for metastatic disease and the Ki67 index was less than $2 \%$. Incidentally, adenomatosis and an additional pNET measuring $2 \mathrm{~mm}$ in size were identified.

\section{Discussion and conclusions}

The $M E N 1$ c.1A > G pathogenic variant was first reported in 2002 [7] in a family with a history of isolated hyperparathyroid hormone levels and hypercalcemia, diagnosed with familial isolated hyperparathyroidism (FIHP). The pathogenic variant segregated with the disease in 3 out of 8 members tested across two generations, while one tested

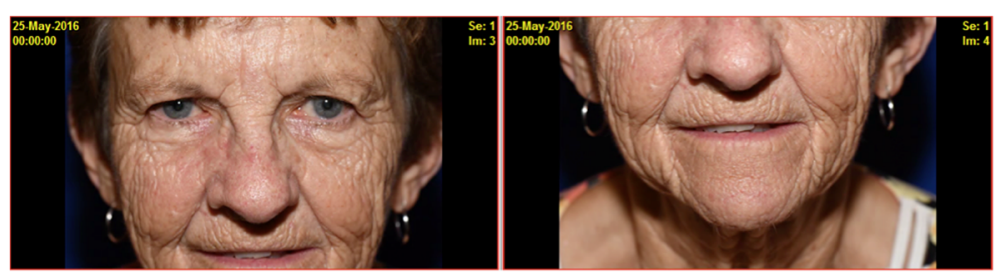

Fig. 176 years old female proband with facial angiofibromas 


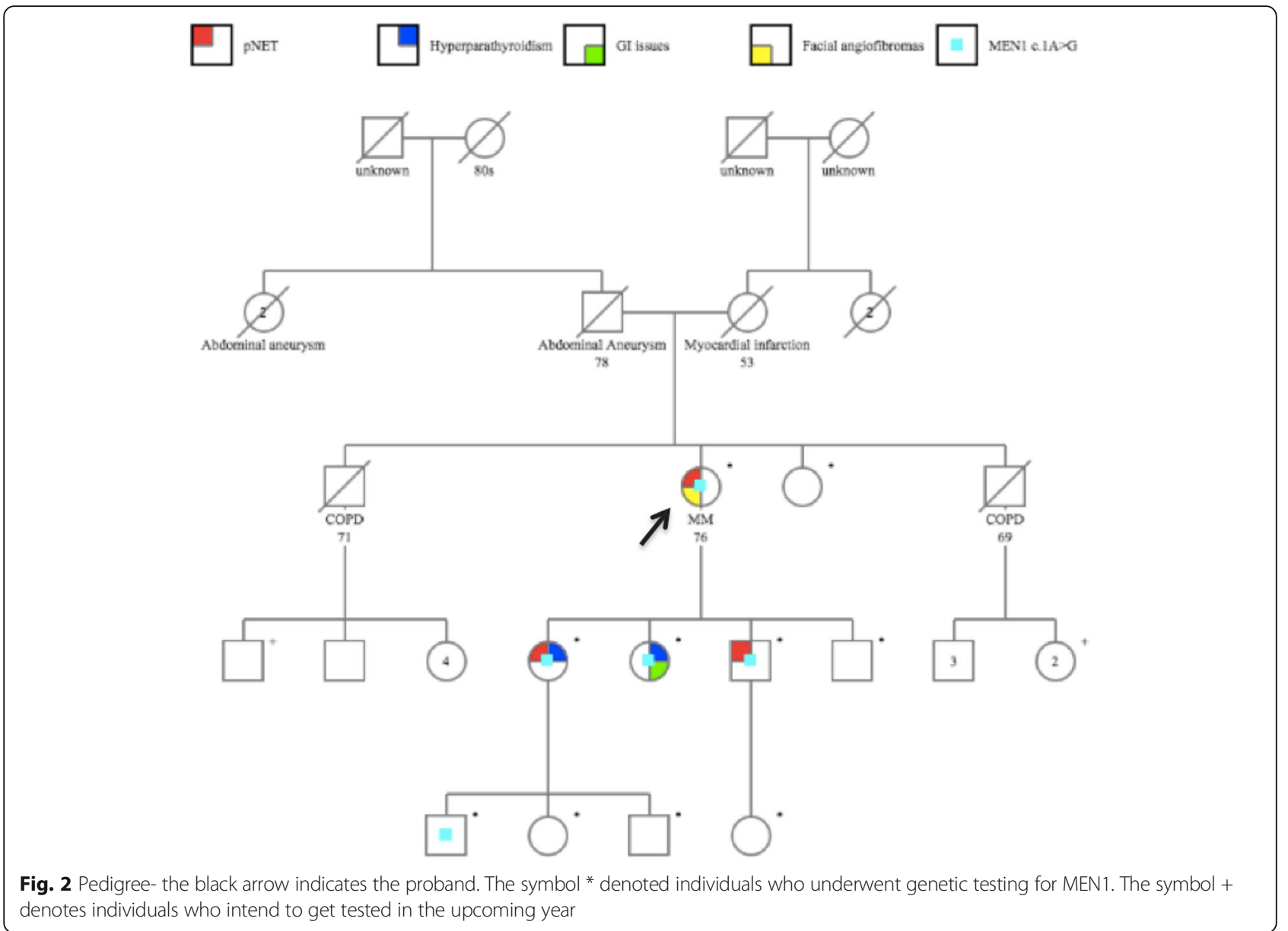

individual had no symptoms and was deemed at risk. Additional literature reports [8] of this pathogenic variant have been published with the affected patients having variable symptoms like parathyroid and pancreatic islet neoplasia with or without other tumors.

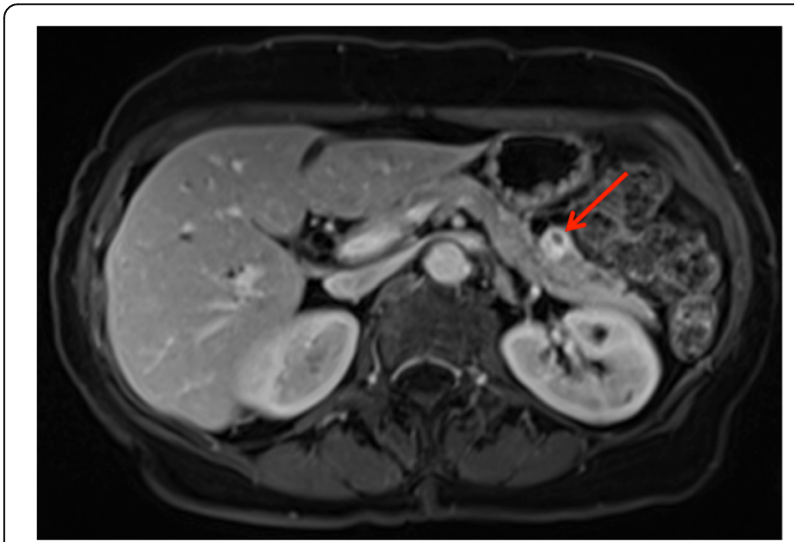

Fig. 3 Axial venous phase MRI showing an enhancing $1.5 \mathrm{~cm}$ cystic mass consistent with pancreatic neuroendocrine tumor in the tail of the pancreas (red arrow)
Other reports involving this start codon of MEN1, such as c.2 $\mathrm{T}>\mathrm{A}$ [9], also exhibit the intrafamilial phenotypic variability associated with variation in this amino acid residue, as different family members with the same pathogenic variant are affected by different endocrine tumors. In our case, the proband was asymptomatic on presentation, despite having two daughters that became symptomatic at the earlier ages of 44 and 48 years. One was diagnosed with hyperparathyroidism and pNET while the other had a diagnosis of hypercalcemia and gastrointestinal symptoms. Both tested positive for the same MEN1 pathogenic variant, which prompted our asymptomatic 76 year-old patient to seek testing. Most patients with MEN1 have shown biochemical manifestations by age 40-50 years [10-12]. Specifically pancreatic neuroendocrine tumors (pNET) are associated with an earlier age of onset in patients with MEN1 mutations than those without MEN1 mutations [10, 12-14]. In our case, the patient did not exhibit any biochemical symptoms of MEN1, in spite of being followed by endocrinology for a history of hypothyroidism and osteoporosis. The pancreatic tumor was found at 76 years of age only after an MRI of the abdomen 
was performed as standard protocol for screening MEN1 family members. This tumor could be correlated as being non-functional given the increased chromogranin A staining.

Regarding the patient's co-existence of MEN1 and lymphoma, there has been a prior case report [15] demonstrating this, and indeed it is an interesting observation. Whilst no known association or mechanism exists in the medical literature, to our knowledge this is only the second case of MEN1 and lymphoma in the same patient. This may be purely co-incidental however given the lack of other cases.

Once our proband tested positive for the pathogenic variant, she was encouraged to share her results with other family members. Her son also tested positive for the pathogenic variant and was found to have a pancreatic tumor on subsequent screening at age 57 years. This highlights the value of medical genetics consultation in patients with MEN1 so that family members at risk can be regularly screened for the endocrine tumors.

This case report brings to attention the variable age of diagnosis for MEN1, the phenotypic heterogeneity of MEN1, even within the same family, and a proband that was asymptomatic until age 76 years, barring facial angiofibromas. The report signifies the importance of medical genetics consultation for diagnosis of MEN1, and, careful screening for MEN1 associated tumors when a pathogenic variant is identified.

\section{Abbreviations}

CHOP: Cyclophosphamide, Hydroxy doxorubicin, Vincristine (Oncovin), and Prednisone; FIHP: familial isolated hyperparathyroidism; IGF-1: Insulin like Growth Factor; MEN1: Multiple Endocrine Neoplasia; PIT: Pituitary Tumor; pNET: Pancreatic Endocrine Tumor; PTH: Parathyroid hormone

\section{Acknowledgements}

We thank the patient and their family for their participation in this report

\section{Funding}

No specific fund has been used for data collection, analyses, results reporting or manuscript writing.

\section{Availability of data and materials}

No new software, databases, application/tool have been described or used: not applicable

\section{Authors' contributions}

CK conception, data collection, writing and revision of the manuscript, PSA: genetics consult, conception, data collection, writing revision and approval of the manuscript, SKM: genetic counseling, writing and revision and approval of the manuscript, JJ: medication, writing and revision and approval of the manuscript, EWK: revision and approval of the manuscript, SLH: Internal medicine consult, revision and approval of the manuscript, JAS: General surgery consult, writing, revision and approval of the manuscript. All authors read and approved the final manuscript.

\section{Consent for publication}

The patient consented and signed on the Mayo Clinic Media authorization form.

\section{Competing interests}

The authors declare that they have no competing interests.

\section{Publisher's Note}

Springer Nature remains neutral with regard to jurisdictional claims in published maps and institutional affiliations.

\section{Author details}

${ }^{1}$ Center for Individualized Medicine, Mayo Clinic, Scottsdale, AZ 85259, USA. ${ }^{2}$ Department of Clinical Genomics, Mayo Clinic, Jacksonville, FL 32224, USA. ${ }^{3}$ Department of Neuroscience, Mayo Clinic, Jacksonville, FL 32224, USA.

${ }^{4}$ Center for Individualized Medicine, Mayo Clinic, Jacksonville, FL 32224, USA. ${ }^{5}$ Department of Health Sciences Research, Mayo Clinic, Division of Biomedical Statistic and Informatics, Rochester, MN 55905, USA. 'Department of Clinical Genomics, Mayo Clinic, Rochester, MN 55905, USA. ${ }^{7}$ Center for Individualized Medicine, Mayo Clinic, Rochester, MN 55905, USA.

${ }^{8}$ Department of Internal Medicine, Mayo Clinic, Jacksonville, FL 32224, USA.

${ }^{9}$ Department of General Surgery, Mayo Clinic, Jacksonville, FL 32224, USA.

Received: 10 January 2017 Accepted: 18 July 2017

Published online: 21 July 2017

References

1. Chandrasekharappa SC, et al. Positional cloning of the gene for multiple endocrine neoplasia-type 1. Science. 1997;276(5311):404-7.

2. Knudson AG. Hereditary cancer: two hits revisited. J Cancer Res Clin. Oncologia. 1996;122(3):135-40.

3. Pannett AA, Thakker RV. Somatic mutations in MEN type 1 tumors, consistent with the Knudson "two-hit" hypothesis. J Clin Endocrinol Metab. 2001;86(9):4371-4

4. Marini F, et al. Multiple endocrine neoplasia type 1. Orphanet J Rare Dis. 2006;: :38.

5. Lemos MC, Thakker RV. Multiple endocrine neoplasia type 1 (MEN1): analysis of 1336 mutations reported in the first decade following identification of the gene. Hum Mutat. 2008;29(1):22-32.

6. de Laat JM, et al. MEN1 redefined, a clinical comparison of mutationpositive and mutation-negative patients. BMC Med. 2016;14(1):182.

7. Villablanca A, et al. Involvement of the MEN1 gene locus in familial isolated hyperparathyroidism. Eur J Endocrinol. 2002;147(3):313-22.

8. Klein RD, et al. Clinical testing for multiple endocrine neoplasia type 1 in a DNA diagnostic laboratory. Genet Med. 2005;7(2):131-8.

9. Remde $\mathrm{H}$, et al. A patient with novel mutations causing MEN1 and hereditary multiple osteochondroma. Endocrinol Diabetes Metab Case Rep. 2015;2015:14-0120.

10. Brandi ML, et al. Guidelines for diagnosis and therapy of MEN type 1 and type 2. J Clin Endocrinol Metab. 2001:86(12):5658-71.

11. Thakker RV. Multiple endocrine neoplasia type 1 (MEN1). Best Pract Res Clin Endocrinol Metab. 2010;24(3):355-70.

12. Machens $\mathrm{A}$, et al. Age-related penetrance of endocrine tumours in multiple endocrine neoplasia type 1 (MEN1): a multicentre study of 258 gene carriers. Clin Endocrinol. 2007:67(4):613-22.

13. Newey PJ, et al. Asymptomatic children with multiple endocrine neoplasia type 1 mutations may harbor nonfunctioning pancreatic neuroendocrine tumors. J Clin Endocrinol Metab. 2009;94(10):3640-6.

14. Triponez F, et al. Epidemiology data on 108 MEN 1 patients from the GTE with isolated nonfunctioning tumors of the pancreas. Ann Surg. 2006;243(2): 265-72.

15. Toshimori H, Okamoto M, Nakatsuru K. Multiple endocrine neoplasia type 1 associated with malignant lymphoma and other complications. Intern Med. 1996;35(11):849-54. 